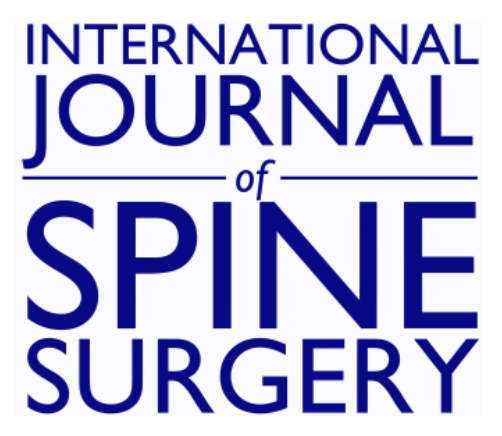

\title{
Defining a Minimum Clinically Important Difference in Patient-Reported Outcome Measures in Lumbar Tubular Microdecompression Patients
}

TRENT A. VANHORN, ZIYAD O. KNIO and TADHG J. O'GARA

Int J Spine Surg 2020, 14 (4) 538-543

doi: https://doi.org/10.14444/7071

http://ijssurgery.com/content/14/4/538

This information is current as of April 25, 2023.

Email Alerts Receive free email-alerts when new articles cite this article. Sign up at: http://ijssurgery.com/alerts 


\title{
Defining a Minimum Clinically Important Difference in Patient-Reported Outcome Measures in Lumbar Tubular Microdecompression Patients
}

\author{
TRENT A. VANHORN, BS, ${ }^{1}$ ZIYAD O. KNIO, MD, ${ }^{1}$ TADHG J. O'GARA, MD ${ }^{1,2}$ \\ ${ }^{1}$ Department of Orthopaedic Surgery, Wake Forest School of Medicine, Winston-Salem, North Carolina, ${ }^{2}$ Department of Neurosurgery, Wake Forest School of \\ Medicine, Winston-Salem, North Carolina
}

\begin{abstract}
Background: Patient-reported outcome measures (PROMs) are critical tools used in the assessment and reporting of surgical outcomes. However, significant differences in PROM scores have not been shown to consistently correlate with clinical improvement from the physician or patient perspective. Defining a minimum clinically important difference (MCID) for PROMs offers interpretation of surgical outcomes with an emphasis on patient-centered feedback. The goal of this study was to define a MCID for the following PROMs in lumbar tubular microdecompression (LTMD) patients: the EuroQol-Five Dimensions (EQ-5D) index, Oswestry Disability Index (ODI), leg pain visual analog scale (VAS), and low back pain VAS.

Methods: This study examined 235 index LTMD patients with PROMs collected at preoperative evaluation and 1year follow-up. Using an anchor-based approach with patient satisfaction index, a receiver operating characteristic analysis was performed to define a MCID in the EQ-5D index, ODI, leg pain VAS, and low back pain VAS.

Results: The patients had a mean age of $65.18 \pm 12.81$ years, and $47.7 \%$ were male. The MCID values for the EQ5D, ODI, leg pain VAS, and low back pain VAS are $0.219,15.0-16.5,0.5$, and $2.5-3.5$, respectively.

Conclusions: This study helps define a MCID for the EQ-5D index in LTMD patients. Given its ease of administration and economic relevance, further characterization of the EQ-5D index may warrant its use as a potential alternative or adjunct to the routinely collected PROMs following spine surgery.
\end{abstract}

Level of Evidence: 3.

Lumbar Spine

Keywords: Oswestry Disability Index, EQ-5D, health state, patient-reported outcome measures

As the trend toward value-based health care continues, patient-reported outcome measures (PROMs) have great value across surgical fields. Optimizing the collection and reporting of PROMs is crucial for improving the efficiency of medical systems collecting these data. For spine surgery patients, the Oswestry Disability Index (ODI), ${ }^{1}$ the Roland-Morris Disability Questionnaire (RM), ${ }^{2}$ and the leg and low back pain visual analog scale $(\mathrm{VAS})^{3}$ are most commonly used. The use of broad health status questionnaires is also pertinent in outcome tracking of spine surgery patients. ${ }^{4}$ At the authors' institution, the EuroQol-Five Dimensions (EQ-5D) Three Level Questionnaire ${ }^{5}$ is routinely collected in addition to the ODI and leg and low back pain VAS in lumbar tubular microdecompression (LTMD) patients. The EQ-5D assesses universal health state and is affordable and easy to understand and can be administered over the phone.
In comparison to disease-specific PROMs, EQ-5D analysis for patients undergoing lumbar spinal surgery has not been well described, although its administration is increasing. ${ }^{6}$

Changes in PROMs are often used to underscore the efficacy of surgical intervention. Unfortunately, statistical significance is not universally associated with patient- or physician-perceived improvement. This discovery has led to increasing interest in defining a minimum clinically important difference (MCID) for PROMs.

Jaeschke et $\mathrm{al}^{7}$ first defined MCID as "the smallest difference in score in the domain of interest which patients perceive as beneficial." Changes to the definition of MCID over the years prompted Chung et $\mathrm{al}^{8}$ to review MCID within the current spine literature. The study describes the fundamental idea of MCID as "a calculated threshold value in 
an outcome of interest that patients and clinicians perceive as clinically meaningful."

MCID ranges have been defined for the following PROMs in lumbar spine surgery patients: ODI, ${ }^{1}$ leg pain VAS, ${ }^{3}$ and low back pain VAS. ${ }^{8,9}$ MCID has not been well defined for the EQ-5D index ${ }^{5,6}$ despite its routine collection in lumbar spinal stenosis patients in order to quantify the value-added of medical and/or surgical management. ${ }^{10}$ The goal of this study was to establish MCID values using an anchor-based method for the EQ-5D index, ODI, leg pain VAS, and low back pain VAS in LTMD patients.

\section{METHODS}

A retrospective review of the senior author's LTMD database was performed. Briefly, this database enrolls patients prospectively before surgery and administers the EQ-5D, ${ }^{5}$ ODI, ${ }^{1}$ and leg and low back pain $\mathrm{VAS}^{3}$ preoperatively and at 1-, 2-, 3-, and 5-year follow-up. Patient satisfaction index (PSI) is also collected at all follow-ups. Patient enrollment began in January 2014 following institutional review board approval of the protocol.

Inclusion criteria for this study were patients $\geq 18$ years of age with symptomatic lumbar spinal stenosis (LSS) who underwent elective index LTMD at least 1 year prior to analysis in July 2019. Patient selection for surgery was based on preoperative evaluation by the senior author and included persistence of neurologic deficits after at least 90 days of conservative nonoperative treatment, absence of instability on radiographs, and preoperative magnetic resonance imaging confirming LSS likely to respond to LTMD. Exclusion criteria included nonindex LTMD cases.

\section{Outcome Measures}

PROMs were collected during office visits or via a standardized telephone script. PSI is a single item, with 4possible responses: (1) "Surgery met my expectations," (2) "Surgery improved my condition enough so that I would go through it again for the same outcome," (3) "Surgery helped me, but I would not go through it again for the same outcome," (4) "I am the same or worse compared to before surgery."

$\mathrm{EQ}-5 \mathrm{D}^{5}$ is a 5 -dimension health state questionnaire assessing mobility, self-care, usual activities, pain/discomfort, and anxiety/depression. Each item scores 1-3, with 3 being the most problematic. Of note, the possible responses are relatively consistent from item to item; that is, a response scoring 1 is generally "I have no problems [performing item,]" a response scoring 2 is generally "I have some problems [performing item]," and a response scoring 3 indicates severe problems. EQ-5D indices were calculated using a validated valuation model for US patient populations, with scores ranging from -0.11 (worse than death) to 1.0 (full health), with 0 indicating a health state comparable to death. ${ }^{11}$

$\mathrm{ODI}^{1}$ is a 10 -item disability questionnaire assessing pain intensity, personal care, lifting, walking, sitting, standing, sleeping, work, social life, and traveling. Each item scores $0-5$, with 5 being the most severe. As ODI is transformed to a composite score on a $0-100$ scale (with 100 being the most severe), the following correction factor was applied in cases where 1 or more ODI questions were left unanswered: ${ }^{12}$

Corrected ODI

$$
=\mathrm{ODI} \times\left(\frac{10}{10-\text { number of missing responses }}\right) .
$$

Leg and low back pain $\mathrm{VAS}^{3}$ items are $0-10$ integer responses to the questions, "On a scale of 0 to 10 , mark your level of leg (low back) discomfort, with 0 being none and 10 being unbearable." No corrections were applied.

\section{Statistical Analysis}

The PSI was dichotomized, with responses 1-3 considered "improved" and response 4 considered "nonimproved." Responses 1-3 identify patients who are able to subjectively appreciate clinical improvement after surgical intervention. Response 4 alone was sufficient for defining "nonimprovement" given that it explicitly captured patients who felt they were the "same or worse" compared to before surgery.

The PSI dichotomization was treated as the anchor in a receiver operating characteristic (ROC) analysis in order to define a MCID in each of 4 PROMs: EQ-5D index, ODI, leg pain VAS, and low back pain VAS. For each of the 4 PROMs, a ROC curve was plotted, an area under the curve was calculated, and an optimal MCID was computed from both the top-left-corner criteria and the Youden index. ${ }^{13}$ All computations and statistical tests were performed in $\mathrm{R}$ ( $\mathrm{R}$ Core Team, Vienna, Austria). The pROC package in $\mathrm{R}$ was used for all components of the ROC analysis. ${ }^{14}$ 
Table. Distribution of metrics captured across visit times.

\begin{tabular}{lcc}
\hline & Preoperative & 1-Year Follow-Up \\
\hline Patient satisfaction index & 0 & 201 \\
EQ-5D index & 201 & 204 \\
ODI & 185 & 192 \\
Leg pain VAS & 211 & 203 \\
Back pain VAS & 204 & 205 \\
\hline
\end{tabular}

Abbreviations: EQ-5D, EuroQol-Five Dimensions; ODI: Oswestry Disability Index; VAS, visual analog scale.

\section{RESULTS}

The senior author's LTMD database included 255 cases performed between January 2014 and July 2018. After excluding 18 revision cases, the remaining sample was comprised of 237 unique index LTMD patients. Only 2 patients $(0.8 \%)$ were lost to follow-up, yielding a final sample of 235 unique index LTMD patients. The patients had a mean age of $65.18 \pm 12.81$ years, and $47.7 \%$ were male. Response rates are presented in the Table.

For the EQ-5D index, the area under the ROC curve was 0.847 (95\% confidence interval: 0.754 0.939). The EQ-5D index MCID was 0.219 for both corner and Youden optimization (Figure 1).

For the ODI, the area under the ROC curve was 0.849 (95\% confidence interval: $0.780-0.917)$. The ODI MCIDs were 15.0 and 16.5 for corner and Youden optimization, respectively (Figure 2).

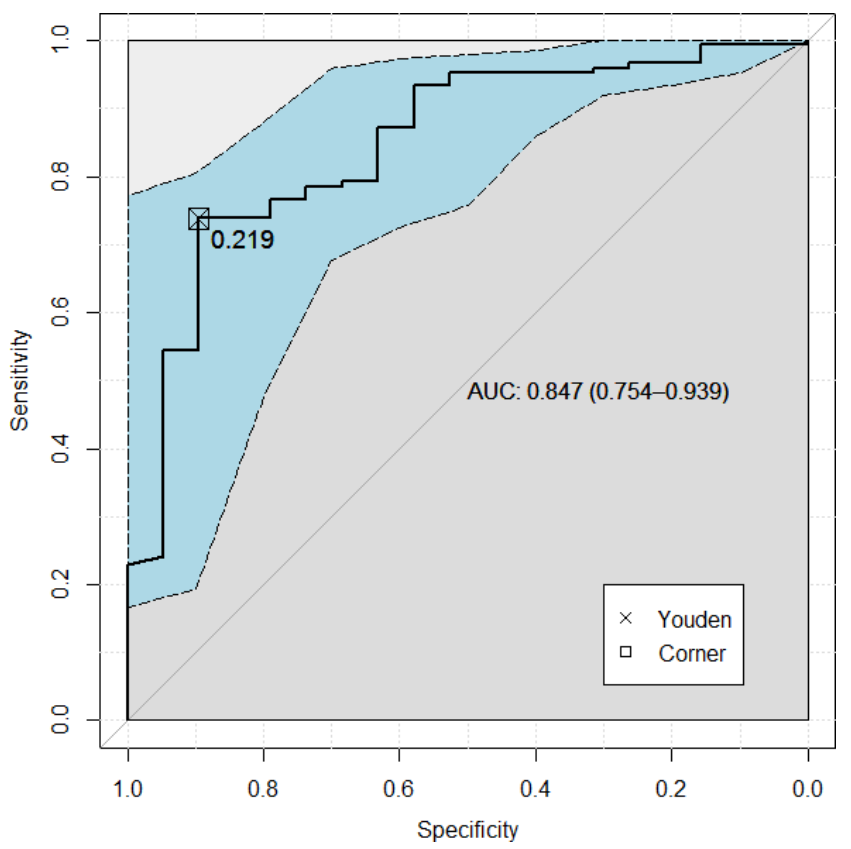

Figure 1. EuroQol-Five Dimensions (EQ-5D) receiver operating characteristic (ROC) curve. The ROC curve for the EQ-5D, taking the patient satisfaction index to be the anchor. The dashed line is a bootstrapped confidence interval. Also reported is the area under the curve and the $95 \%$ confidence interval and the defined minimum clinically important difference thresholds.

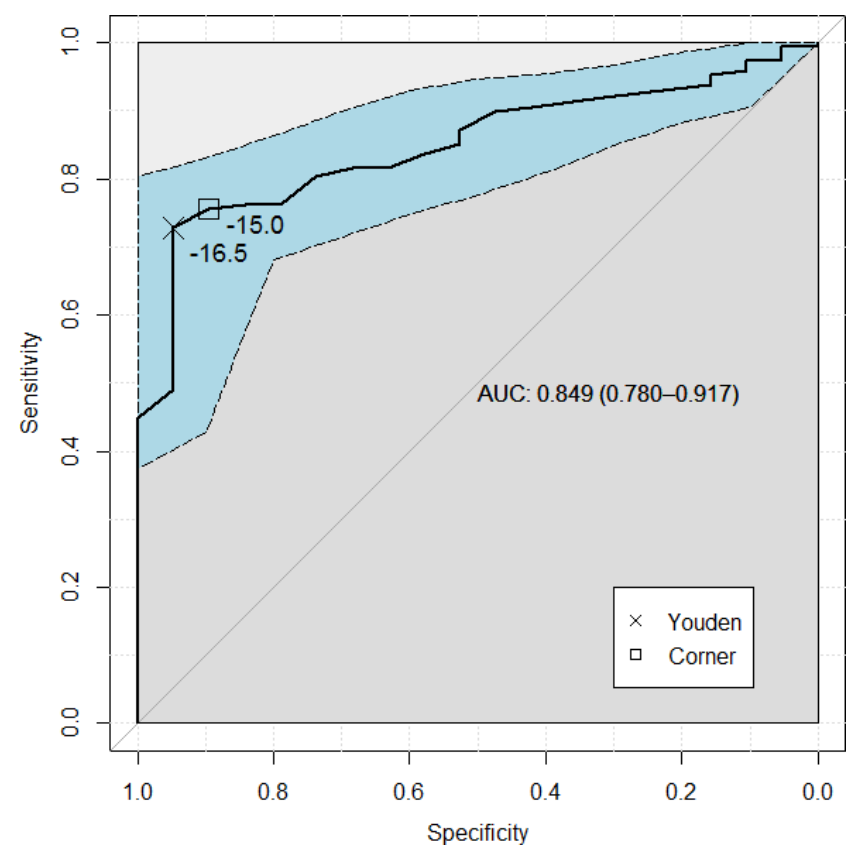

Figure 2. Oswestry Disability Index (ODI) receiver operating characteristic (ROC) curve. The ROC curve for the ODI, taking the patient satisfaction index to be the anchor. The dashed line is a bootstrapped confidence interval. Also reported is the area under the curve and the $95 \%$ confidence interval and the defined minimum clinically important difference thresholds.

For the leg pain VAS, the area under the ROC curve was $0.885(95 \%$ confidence interval: $0.822-$ 0.949). The leg pain VAS MCID was 0.5 for both corner and Youden optimization (Figure 3).

For the low back pain VAS, the area under the ROC curve was 0.717 (95\% confidence interval: $0.623-0.812$ ). The low back pain VAS MCIDs were 2.5 and 3.5 for corner and Youden optimization, respectively (Figure 4).

\section{DISCUSSION}

This study has defined a MCID of 0.219 for the EQ-5D index in LTMD patients. A MCID for the EQ-5D index has not been well defined previously. Several studies have contributed to a wide range of $0.03-0.52 .{ }^{6}$ In patients with chronic low back pain and degenerative disc disease, 1 proposed MCID for EQ-5D index was $0.173 .{ }^{15}$ The presently described EQ-5D index MCID serves as a guide for meaningful score interpretation. As the MCID for the EQ-5D index evolves and the range for specific procedures narrows, it may become an increasingly valuable tool in the design of clinical trials and in critical evaluation of study results. The MCID value of $15.0-16.5$ for the ODI is comparable to previously defined MCID values of $12.8^{9}$ or $12.88 .{ }^{15}$ The MCID value of 0.5 for the leg pain 


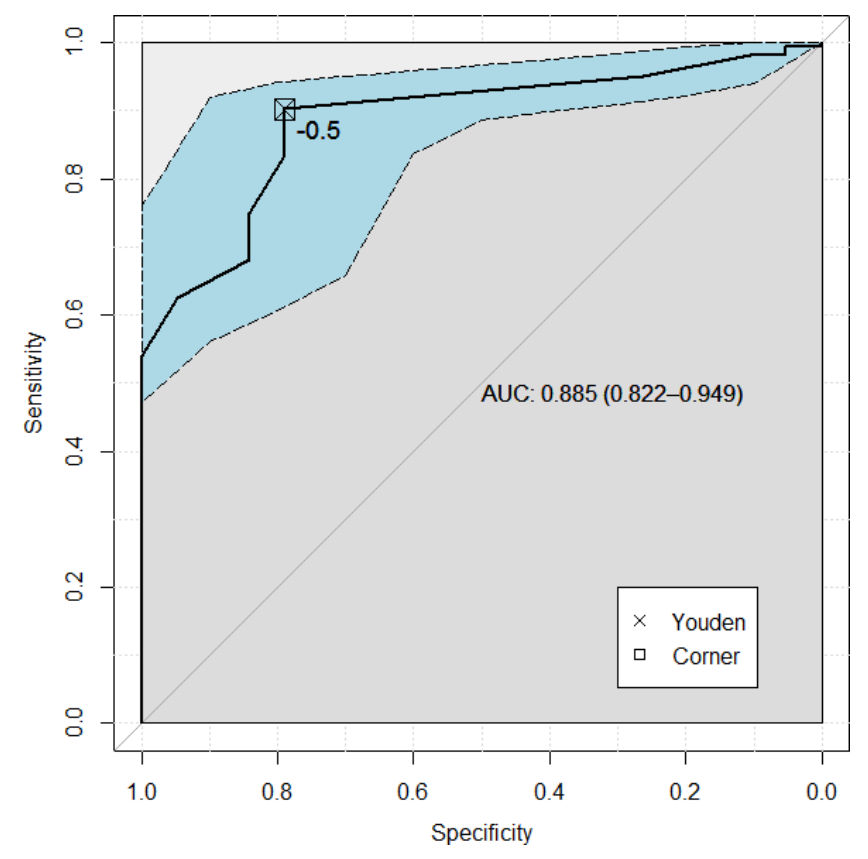

Figure 3. Leg pain visual analog scale (VAS) receiver operating characteristic (ROC) curve. The ROC curve for the leg pain VAS, taking the patient satisfaction index to be the anchor. The dashed line is a bootstrapped confidence interval. Also reported is the area under the curve and the $95 \%$ confidence interval and the defined minimum clinically important difference thresholds.

VAS is less than a previously defined MCID value of 1.6. ${ }^{9}$ The MCID value of 2.5-3.5 for the low back pain VAS is greater than the commonly cited MCID value of 1.2. ${ }^{9}$

When compared to previously defined MCID thresholds in patients with low back pain, this study's results suggest that a MCID may not necessarily be considered a static value when applied to PROMs. ${ }^{16}$ The expanding compilation of proposed values lends itself to the use of MCID ranges for different purposes. For instance, providers may tailor the use of MCID with respect to treatment risk. In the setting of a low-risk intervention, achieving a value on the lower end of the MCID range might be acceptable, while a more stringent threshold at the higher end of a range should be considered for higher-risk effects.

Information provided by overall health questionnaires such as the EQ-5D index in surgical patients is at a premium, as systems operate within a value-based system. This PROM incorporates patients' judgments about their own health status and indicates which health states are deemed more desirable than others. Compared to disease-specific PROMs, routine collection of the EQ-5D provides a means to compare treatment efficacy across a multitude of therapeutic domains. From an economic valuation standpoint,

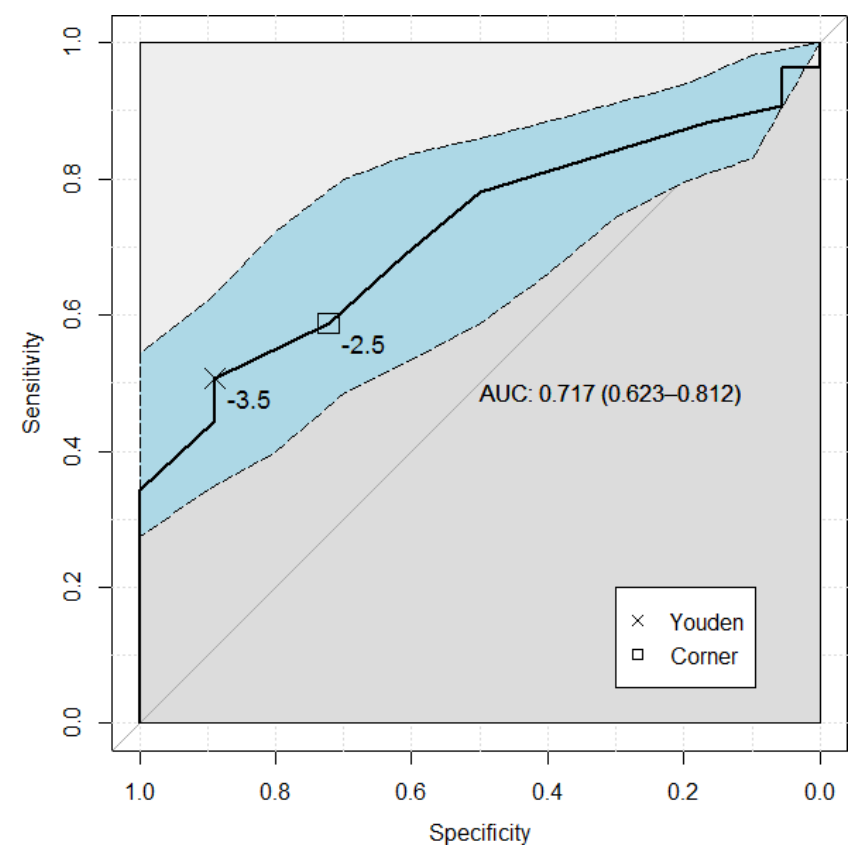

Figure 4. Low back pain visual analog scale (VAS) receiver operating characteristic (ROC) curve. The ROC curve for the low back pain VAS, taking the patient satisfaction index to be the anchor. The dashed line is a bootstrapped confidence interval. Also reported is the area under the curve and the $95 \%$ confidence interval and the defined minimum clinically important difference thresholds.

optimal analysis of EQ-5D indices may inform policy and practice decision making. For these reasons, strengthening the current landscape of MCID for the EQ-5D has major implications.

Different methods for calculating and reporting MCID values have been defined. ${ }^{8}$ An anchor-based approach is most often cited and preferred when compared to a distribution-based approach. ${ }^{8}$ In this approach, the PROM of interest is paired, or "anchored," to another measure of the patient's change in condition. The changes in scores on the PROM are then compared to the changes in score of the chosen anchor, and statistical tools are applied to determine MCID. Despite a lack of standardization surrounding MCID calculation, overall reporting and desirability have increased in recent years. ${ }^{6,8,16}$ The authors recognize that the PSI anchor, dichotomized by response, was not a validated assessment of clinical improvement; however, the methodology was consistent with several existing studies using similar subjective anchors. ${ }^{9,15}$ Copay et $\mathrm{al}^{9}$ found that a satisfaction-with-results scale was better associated with PROM change scores compared to the health transition item found on the Short Form of the Medical Outcomes Study (SF-36). A satisfaction index is inherently subjective and patient centered. 
Evaluation of surgical intervention within an increasingly value-based system warrants significant inclusion of these characteristics. The current landscape supports the use of a satisfaction index in MCID determination. Preoperative counseling and expectation discussions become important considerations given the potential impact on postoperative satisfaction. MCID may serve as an aid in the preoperative discussion related to reasonable expectations of the surgery.

The present study is limited mainly by a narrowly defined patient population and response. By including only LTMD patients in this study, it is possible that the findings can be generalized only to LTMD patients. MCID variations based on diagnosis or procedure should be considered if the patients' perception of change is inherently different from others. ${ }^{8}$ To our knowledge, a divergence in perception of meaningful change has not been described. While follow-up rate was not the most limiting factor (235 of 237 index LTMD patients), response rates at each visit time were suboptimal. This is just as much a limitation as it is strong support for the need to define a MCID for various PROMs, as each questionnaire is associated with a collection burden. Favorable characteristics of overall health questionnaires such as the EQ-5D index reveal an opportunity to improve efficiency related to PROM collection while simultaneously engaging in rigorous analysis of surgical intervention. Mueller et $\mathrm{al}^{4}$ found that the EQ-5D correlated well with the ODI and other PROMs for surgical spine patients. Kovacs et $\mathrm{al}^{17}$ responded to the proposed correlation by stating that compared to other PROMs, the EQ-5D is not as responsive to clinical changes.

The authors plan to investigate patient and surgical characteristics associated with false-positive and false-negative responses under the defined MCIDs. Identification of independent predictors for patient-perceived clinical improvement following LTMD is a novel endeavor. Examination of falsepositive and false-negative results may also enable us to tailor our recommendations and counseling to individual patients, accounting for differences in preoperative questionnaire scores. Additionally, we plan to compare our proposed MCID with any values that appear in the emerging literature.

\section{CONCLUSIONS}

This study offers a MCID for the EQ-5D index in LTMD patients. Given its ease of administration and economic relevancy, further characterization of the EQ-5D may warrant its use as a potential alternative or adjunct to the routinely collected PROMs following spine surgery.

\section{ACKNOWLEDGMENTS}

The authors would like to thank Wendy Williams and Marcy Lewis, RN, for their assistance in outcomes collection.

\section{REFERENCES}

1. Fairbank JC, Pynsent PB. The Oswestry Disability Index. Spine. 2000;25(22):2940-2952; discussion 2952.

2. Roland M, Morris R. A study of the natural history of low-back pain. Part II: development of guidelines for trials of treatment in primary care. Spine. 1983;8(2):145-150.

3. McCaffery M, Beebe A. Pain: Clinical Manual for Nursing Practice. Baltimore, MD: Mosby; 1993.

4. Mueller B, Carreon LY, Glassman SD. Comparison of the EuroQOL-5D with the Oswestry Disability Index, back and leg pain scores in patients with degenerative lumbar spine pathology. Spine. 2013;38(9):757-761.

5. The EuroQol Group. EuroQol-a new facility for the measurement of health-related quality of life. Health Policy (Amsterdam, Netherlands). 1990;16(3):199-208.

6. Coretti S, Ruggeri M, McNamee P. The minimum clinically important difference for EQ-5D index: a critical review. Expert Rev. Pharmacoecon Outcomes Res. 2014;14(2):221-233.

7. Jaeschke R, Singer J, Guyatt GH. Measurement of health status. Ascertaining the minimal clinically important difference. Control Clin Trials. 1989;10(4):407-415.

8. Chung AS, Copay AG, Olmscheid N, Campbell D, Walker JB, Chutkan N. Minimum clinically important difference: current trends in the spine literature. Spine. 2017;42(14):1096-1105.

9. Copay AG, Glassman SD, Subach BR, Berven S, Schuler TC, Carreon LY. Minimum clinically important difference in lumbar spine surgery patients: a choice of methods using the Oswestry Disability Index, Medical Outcomes Study questionnaire Short Form 36, and pain scales. Spine J. 2008;8(6):968974.

10. Weinstein JN, Tosteson TD, Lurie JD, et al. Surgical versus nonsurgical therapy for lumbar spinal stenosis. $N$ Engl $J$ Med. 2008;358(8):794-810.

11. Shaw JW, Johnson JA, Coons SJ. US valuation of the EQ-5D health states: development and testing of the D1 valuation model. Med Care. 2005;43(3):203-220.

12. Mehra A, Baker D, Disney S, Pynsent PB. Oswestry Disability Index scoring made easy. Ann $R$ Coll Surg Engl. 2008;90(6):497-499.

13. Unal I. Defining an optimal cut-point value in ROC analysis: an alternative approach. Comput Math Methods Med. 2017;2017:3762651-3762614.

14. Robin X, Turck N, Hainard A, et al. pROC: an opensource package for $\mathrm{R}$ and $\mathrm{S}+$ to analyze and compare $\mathrm{ROC}$ curves. BMC Bioinformatics. 2011;12(1):77. 
15. Johnsen LG, Hellum C, Nygaard OP, et al. Comparison of the SF6D, the EQ5D, and the Oswestry disability index in patients with chronic low back pain and degenerative disc disease. BMC Musculoskelet Disord. 2013;14:148.

16. Hung M, Saltzman CL, Kendall R, et al. What are the MCIDs for PROMIS, NDI, and ODI instruments among patients with spinal conditions? Clinical orthopaedics and related research. Clin Orthop. 2018;476(10):2027-2036.

17. Kovacs FM, Royuela A, Zamora J, Abraira V. Re: Mueller B, Carreon LY, Glassman SD. Comparison of the EuroQol-5D with the Oswestry disability index, back and leg pain scores in patients with degenerative lumbar spine pathology. Spine 2013;38:757-61. Spine. 2013;38(17):1523.

Disclosures and COI: This research did not receive any specific grant from funding agencies in the public, commercial, or not-for-profit sectors. The authors report no conflicts of interest.

Corresponding Author: Tadhg J. O'Gara, MD, Department of Orthopaedic Surgery, Wake Forest School of Medicine, 1 Medical Center Boulevard, Winston-Salem, NC 27157. Phone: (336) 716-3950; Email: togara@wakehealth.edu.

Published 28 August 2020

This manuscript is generously published free of charge by ISASS, the International Society for the Advancement of Spine Surgery. Copyright (C) 2020 ISASS. To see more or order reprints or permissions, see http://ijssurgery.com. 